Cinémas

Revue d'études cinématographiques

Journal of Film Studies

\title{
Vitesse et dématérialisation. Le corps du toon chez Tex Avery
}

\section{Johanne Villeneuve}

Volume 7, numéro 1-2, automne 1996

La Représentation du corps au cinéma

URI : https://id.erudit.org/iderudit/1000932ar

DOI : https://doi.org/10.7202/1000932ar

Aller au sommaire du numéro

Éditeur(s)

Cinémas

ISSN

1181-6945 (imprimé)

1705-6500 (numérique)

Découvrir la revue

Citer cet article

Villeneuve, J. (1996). Vitesse et dématérialisation. Le corps du toon chez Tex Avery. Cinémas, 7(1-2), 55-72. https://doi.org/10.7202/1000932ar

\section{Résumé de l'article}

Il s'agit d'interroger la signification de l'oeuvre d'animation de Tex Avery en rapport avec une certaine lecture du corps comique et de la matérialité. Héritier à la fois de la tradition burlesque européenne et de l'archétype du trickster, inspiré par les héros " économiques " du XVIII ${ }^{\mathrm{e}}$ siècle, le toon propose toutefois l'exubérance dans la négation, la vitesse par l'explosion matérielle et la compulsion d'un corps sans cesse renouvelable : flexibilité et adaptabilité fantastiques, profusion des moyens d'action, etc. La prédation et la dévoration sont au coeur de cette agitation qui mobilise le toon, mais elles s'accompagnent toujours de la capacité du corps à faire éclore l’univers tout entier. 


\section{Vitesse et dématérialisation. Le corps du toon chez Tex Avery}

\section{Johanne Villeneuve}

\section{RÉSUMÉ}

Il s'agit d'interroger la signification de l'œuvre d'animation de Tex Avery en rapport avec une certaine lecture du corps comique et de la matérialité. Héritier à la fois de la tradition burlesque européenne et de l'archétype du trickster, inspiré par les héros "économiques" du $\mathrm{XVIII}$ siècle, le toon propose toutefois l'exubérance dans la négation, la vitesse par l'explosion matérielle et la compulsion d'un corps sans cesse renouvelable: flexibilité et adaptabilité fantastiques, profusion des moyens d'action, etc. La prédation et la dévoration sont au cœur de cette agitation qui mobilise le toon, mais elles s'accompagnent toujours de la capacité du corps à faire éclore l'univers tout entier.

\section{ABSTRACT}

This inquiry into the significance of Tex Avery's work in animation situates it within a specific reading of the comic body and its materiality. Heir to both the European burlesque tradition and the archetype of the trickster, inspired by the "economic" heroes of the eighteenth century, the "toon" nevertheless projects its exuberance through negation, its speed through material explosion and the momentum of an endlessly renewable body, enjoying fantastic flexibility and adaptability, with a profusion of means of action, etc. Predation and devouring are key to the frenetic activity of the toon, but they always go hand in hand with the body's ability to open up the whole universe. 
C'est au milieu des années trente, au moment où la manière Disney s'était déjà fortement imposée dans le cinéma d'animation, que Tex Avery et ses collaborateurs ont commencé à infléchir le genre, à inventer pour ainsi dire le cartoon tel que nous le connaissons et ne cessons de le reconnaître à travers ses influences nombreuses, avec ses pointes satiriques et son sens de la dérision, son rythme débridé et ses dérives burlesques. D'abord à la Warner à partir de 1936 (les séries de Porky Pig, Daffy Duck, Egghead, Bugs Bunny and Elmer Fudd), puis chez MGM dans les années quarante et cinquante (les séries mettant en vedette les célèbres loups de Avery, celles également de Screwy Squirrel, Droopy, Spike), Avery a contribué à inventer un genre. C'est aussi à son art de la parodie qu'il doit sa réputation, pour avoir détourné l'imagerie enfantine et enchantée de Disney vers une féroce mise en représentation des fantasmagories adultes et des rôles sociaux. Mais si l'œuvre peut être parfois prise pour un "hommage" à Disney - Disney perverti, machinique, livré cru - la cible de la parodie déborde cependant largement le cadre du cinéma d'animation. Le style Avery bouffe de tout: on se souviendra de Little Rural Riding Hood (1949) parodiant Le Petit Chaperon rouge et Le Rat des villes et le Rat des champs tout à la fois, de Swing Shift Cinderella (1945), de Uncle Tom's Cabana (1947) et de la série George and Junior (1947-1948) inspiré du couple de travailleurs saisonniers dans Of Mice and Men (John Steinbeck, 1937).

Il y aurait beaucoup à dire sur l'œuvre de Avery, mais je me contenterai simplement d'interroger sa signification en rapport avec une certaine lecture du corps comique et de la matérialité. Le cartoon, issu à la fois de l'art du dessin et de la technologie cinématographique, propose un regard nouveau sur le corps et son rapport à la matérialité filmique; au-delà de la standardisation et de la commercialisation des films d'animation, interroger cette nouveauté, sonder ce regard pour tenter de voir ce qu'il voit, c'est faire glisser dans une perspective nouvelle les intrigues narratives, les ruses animalières du trickster et la théâtralité burlesque.

La mécanique du cartoon et le burlesque existentiel à la Keaton, dont les ramifications s'étendent jusqu’à la comédie 


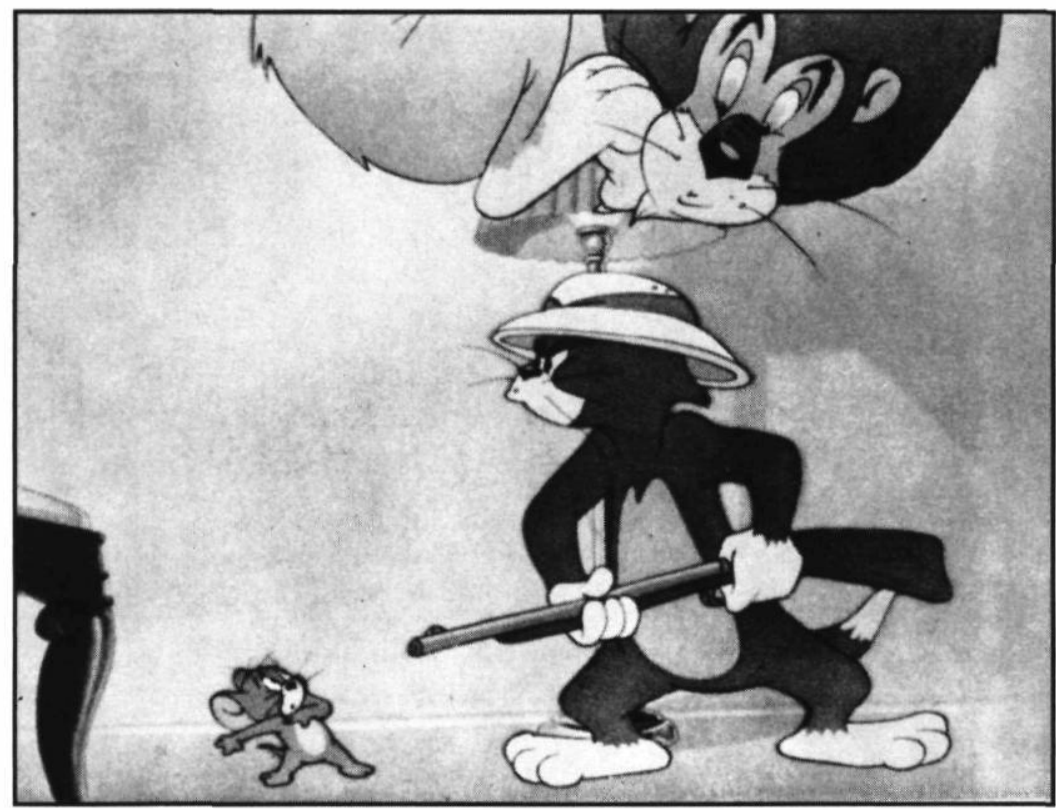

Tom and Jerry de Tex Avery

Collection Cinémathèque québécoise

absurde d'un Beckett, sont certes liés par une obsession commune du corps. Mais quelque chose les distinguent dans leurs effets et la nature du discours qu'ils tiennent sur le monde: si le burlesque existentiel met l'emphase sur le piétinement de l'individu solitaire aux portes d'un jeu social dont il ne peut que mimer la gestuelle, le burlesque d'animation insiste au contraire sur la persévérance machinique du jeu social, sur l'absorption de l'individu par la mécanique sociale et ses jeux d'interaction. Héritier des chassés-croisés de la tradition burlesque et du vaudeville, le toon appartient à un engrenage de l'action qui ne connaît pas l'essoufflement nihiliste, mais plutôt l'exubérance dans la négation: destruction du corps du toon, remise en place, négation de la destruction elle-même, répétition de la destruction par tous les moyens. Ces moyens n'ont pas de limite: la chasse, la poursuite, la dévoration, la mise à sac, l'explosion, l'implosion, l'émulsion, la décollation, la mutilation, la propulsion et la pulvérisation. Tous ces réseaux d'activités concourent à faire du toon le représentant exacerbé des jeux de pouvoir 
sociaux, tantôt victime, tantôt champion de ces jeux. Quoi qu'il en soit, le burlesque n'arrive jamais ici à l'épuisement de l'être, à cette infinitésimalité de l'intrigue, quand ce n'est aux dernières ressources de l'apraxie, comme c'est le cas dans le burlesque existentiel. Si le cas du toon constitue lui aussi un cas paroxystique où la négativité est poussée à l'extrême limite, c'est au sens où l'action ne cesse de rejaillir au cœur même de la négation. Là où la lenteur contribue chez Beckett à repousser sans cesse cette limite, la vitesse fait apparaître dans le cartoon, de manière presque stroboscopique, l'immense capacité du mouvement à se régénérer.

Chez Tex Avery, tout particulièrement, la mécanique sociale est à la fois démontée et poussée aux extrêmes. Les toons, bien sûr, sont issus des mondes objectaux burlesques, de la conception de la péripétie basée sur l'accidentel, la méprise sans cesse recouvrable et le jeu des échanges sociaux. La victime devient souvent le bourreau, le chasseur se voit souvent pris en chasse par sa proie. Renversement carnavalesque? Retournement utopique? Au plus près, c'est du côté de la littérature à intrigues qu'il faut chercher. Des aléas bien connus de la vertu et de la résistance, dont cette littérature s'est faite la médiatrice par excellence aux XVII et XVIII ${ }^{e}$ siècles, le toon a conservé plusieurs traits, mais les a systémiquement exacerbés. Les subtilités des jeux de cour, celles de la cour amoureuse, la volonté de contrôler les affects, le sens de la compétitivité, la pensée stratégique et le calcul de ses chances dans le cercle mondain sont au nombre des modalités de l'intrigue qui prévalent dans les univers fictionnels des $\mathrm{XVII}^{\mathrm{e}}$ et XVIII ${ }^{\mathrm{e}}$ siècles. Le cartoon va reprendre la recette à la base; il va dépouiller en quelque sorte ces univers maintenant stéréotypés en conservant la logique structurelle du désir et des relations sociales, mais en ajoutant cependant quelque chose de déterminant: l'intensité et le parodique. Comme le burlesque l'avait lui-même fait au XVII siècle en transposant les jeux de pouvoirs sur la mécanique simpliste de la bourla (la farce), le cartoon assume pleinement la simplicité des rapports sociaux qu'il transpose, cette fois, sur un régime de vitesse sans précédent ; chez Avery, la logique des stratégies de pouvoir et des rapports sociaux acquiert donc un caractère explosif. Les structures 
simples des activités intrigantes passent par l'exubérance et la pure fantasmagorie, par une explosion des désirs, voire de besoins primaires: manger, accroître son pouvoir ou son profit, se débarrasser d'autrui, etc. deviennent les prétextes à la production de la vitesse. Le caractère exponentiel de l'activité "toonesque" devient ainsi le moteur même de l'intrigue.

Source lointaine mais entièrement reprise et revivifiée par le cartoon, la mécanique burlesque du roman bourgeois met en scène un corps constamment pris dans le tourbillon des gaffes et des méprises. Ce corps-mouvement, dont le mouvement même - quand ce n'est l'inertie soudaine et la flagrante mésadaptation au monde - subit les contraintes désastreuses mais néanmoins drôlatiques de vitesses imprévisibles, s'adonne à l'effet domino, à la surenchère et la répétition des erreurs. C'est la comédie des erreurs poussée jusqu'à ses délimitations abstraites, mathématiques, quasi pures. Ce corps comique est irréductible à un complexe identitaire telle la "personnalité héroïque" ou la "figure tragique". Bien sûr, tout récit suppose, pour le dire comme Aristote, que le caractère soit déterminé par l'action; c'est là la condition première de l'ethos dans la poétique. Et l'on sait toutefois que la littérature moderne occidentale, particulièrement depuis le XVIII ${ }^{e}$ siècle, a privilégié le personnage au détriment de l'action entendue au sens aristotélicien. Or la comédie burlesque offre pour ainsi dire un autre amalgame, un autre rapport entre l'action et le personnage. Refusant de placer le personnage au-dessus de l'action, la comédie burlesque privilégie un aspect du personnage dans sa confrontation directe avec l'action: son "corps", ou pour l'énoncer plus clairement, le jeu qui met aux prises un corps qui se veut sujet et un monde qui ne cherche qu'à objectiver ce corps, qu'à le rendre conforme à son rythme et à sa mesure. Ce monde, lui-même fait d'objets, ne cesse d'apparaître en "sujet" de l'action, investi d'une volonté et d'une intentionnalité qui échappent d'autant plus à l'entendement du héros que celui-ci accuse constamment du retard par rapport à l'activité mise en train, quand il ne se précipite pas malgré lui au-devant des choses. En fait, les régimes de vitesse entre les objets (le héros et ce qui l'entoure) ne concordent pas ou coïncident par pur improbable. Le corps devient central au 
sein de cette mécanique des ratés parce qu'il est immédiatement saisissable, immédiatement visible et sensible dans l'espace social.

Les lecteurs du Roman bourgeois de Furetière ne seront pas surpris d'y trouver des scènes "toonesques" avant l'heure, comme celle-ci où Nicodème cherche maladroitement à prendre congé d'une "bonne famille » dont il souhaite épouser la fille :

Il ne fut pas assez hardy pour saluer, en sortant, sa maistresse de la maniere qu'il est permis aux amants declarez. Pour Javotte, elle se contenta de luy faire une reverence muette; mais en se levant elle laissa tomber un peleton de fil et ses ciseaux, qui estoient sur sa juppe. Nicodeme se jette aussitost avec precipitation a ses pieds pour les relever; Javotte se baisse, de son coste, pour le prevenir; et, se relevant tous deux en mesme temps, leurs deux fronts se heurterent avec telle violence, qu'ils se firent chacun une bosse. Nicodeme, au desespoir de ce malheur, voulut se retirer promptement; mais il ne prit pas garde a un buffet boiteux qui estoit derriere luy, qu'il choqua si rudement qu'il en fit tomber une belle porcelaine, qui estoit une fille unique fort estimee dans la maison. La-dessus, la mere esclatte en injures contre luy. Il fait mille excuses, et en veut ramasser les morceaux pour en renvoyer une pareille; mais en marchant brusquement avec des souliers neufs sur un plancher bien frotté et tel qu'il devoit pour des fiançailles, le pied luy glissa, et comme, en ces occasions, on tâche à se retenir à ce qu'on trouve, il se prit aux houpes des cordons qui tenoient le miroir attaché; or, le poids de son corps les ayant rompus, Nicodeme et le miroir tomberent en même temps. [...] La procureuse, s'écriant plus fort qu'auparavant, luy dit: "Qui m’a amené ici ce ruinemaisons, ce brise-tout?" et se met en estat de le chasser avec le manche du ballay. Nicodeme, tout honteux, gagne la porte de la salle; mais, estant en colere, il l'ouvrit avec tant de violence, qu'elle alla donner contre un theorbe qu'un voisin avait laisse contre la muraille, qui fut entierement brisée [...].

Le corps comique est un corps en trop qui se cogne, choque et scandalise, un corps hors-norme dont le comportement excentrique fait se briser la limite des convenances. C'est un 
corps qui se démonte, qui n'arrive pas à trouver sa place, celui d'une individualité à la constante recherche d'une position sociale. Mais c'est aussi un corps qui prend trop de place et fait s'écrouler l'ordre ambiant, un corps vorace qui absorbe en lui toutes les contradictions du social et les exprime dans une pantomime loufoque. Rôle, action et personnalité sont des catégories qui se fondent en une seule chorégraphie.

Comme le propose Olivier Mongin, en réévaluant les catégories deleuziennes ("Les métamorphoses du corps comique", 1993), le corps comique ne saurait se voir confiner, soit du côté de l'image-mouvement, soit du côté de l'image-temps; car il s'agit bien d'un corps "[...] qui se trouve pris dans un double rythme: celui du mouvement et celui du temps", celui de la matière et du mécanique :

Comme si le mouvement impossible favorisait le passage, le coup d'éclat du temps, comme si la courbure des corps comiques échappait à l'oscillation entre le corps mis en mouvement, l'ébranlement et la captation, le ravissement par le temps. Le comique: cette "incarnation " indéfiniment réitérée qui lutte contre une double désincorporation, celle d'un corps à corps du temps et du mouvement (Mongin, p. 127).

Ce que Longin applique au corps comique de Tati ou de Jerry Lewis est d'autant plus vrai pour le corps du toon. Car la matérialité du toon est pleinement incorporée par la matière ambiante; et celle-ci est indissociable du filmique. Dans l'univers "toonesque", rien ne sépare le vivant du mort - ce qui rend impossible la mort elle-même - et rien ne permet de distinguer entre le vivant et les choses. Plus encore: l'univers " toonesque" repose sur la continuité parfaite entre la matérialité et l'immatériel, puisque tout peut y apparaître à tout instant, comme tout peut y être dissous. Même les "trous" peuvent être "déplacés", mutés en objets que les personnages manipulent au gré des stratégies dont ils usent pour se jouer les uns des autres '.

Tex Avery reprend donc le corps comique de la tradition burlesque pour l'insérer dans le contexte des désirs primaires du toon. Ses personnages sont anti-héroïques, au sens d'un renversement de l'héroïsme romanesque; ils sont à la fois déliés, lâchés, 
incontôlables, spontanés, et pourtant entraînés malgré eux dans un décor primaire où les choses sont toujours crûment exposées aux seules fins de la spectature. Ils sont soumis à l'action, conçus en fonction d'une intrigue structurellement simple. Mais une certaine complexité réside malgré tout dans la capacité du toon à créer diversion par rapport à la simplicité de la trame narrative. Plutôt que de l'" action" du récit, il faudrait parler ici d' "activités" où le rythme, la vitesse et la répétition viennent déjouer une intrigue souvent attendue, en tout les cas prévisible. Ces activités "toonesques" ne signifient pas pour autant la mort de l'intrigue, ou sa dissolution, bien au contraire, puisque tout ce qui fait l'intrigue moderne s'y trouve exacerbé, amené aux pulsations les plus rapides des désirs intrigants. Pour user encore des termes deleuziens, nous dirons qu'Avery fait coïncider le geste avec l'intrigue. Si selon Deleuze le gestus défait l'intrigue pour "[...] remettre le temps dans le corps", pour réduire le personnage " [...] à ses propres attitudes corporelles ", à sa théâtralisation (p. 250), force est de constater combien chez Avery l'intrigue n'est autre chose qu'une modalité d'un corps rivé à la plénitude de ses désirs et de ses appréhensions et, inversement, combien le corps est entièrement investi dans l'immatérialité de la vitesse, soit capable de se désintégrer et de se réinstituer selon le bon plaisir du dessinateur.

Cette forme de distanciation par laquelle les toons s'accommodent de leur statut purement filmique s'observe fréquemment chez Avery: les toons s'adressent aux spectateurs, exhibent le caratère aléatoire et éphémère de leur existence Le sort qui guette sans cesse la matérialité ludique de leur corps qui se voit fréquemment dupliqué, percé, aplati, gonflé, étiré, fragmenté, accentue cette distanciation. C'est dans cette mesure que l'intrigue, le corps et le filmique n'appartiennent qu'à une seule fabrique. Les toons reconnaissent eux-mêmes le caractère artificiel de leur fabrication; ils sont appelés à parodier le rôle des stars davantage qu'ils ne jouent des personnages. Porky, Droopy, Daffy Duck se retrouvent dans des décors en tout genre (western, policier, bucolique), dans des rôles divers, à jouer des aventures, sur le modèle des vedettes d'Hollywood. Comme les stars, ils sont immortels, le savent et le font valoir. En parodiant 
Hollywood, Avery donne au désir de dévoration si présent dans ses films une connotation économique importante: la dévoration du toon, c'est aussi celle à laquelle pousse le système basé sur la production en série ${ }^{2}$.

La dévoration, la prédation et la compétitivité conduisent dans l'univers "toonesque " à la folle expansion du mouvement. C'est la vitesse qui prend en charge la complexité d'une psychopathologie sociale: vitesse de la poursuite, convulsions de l'image et du corps, hystérie, paranoïa, convoitise, excitation lubrique dont l'outrance déborde souvent l'entendement et déclenche le rire. La vitesse devient le mode d'actualisation des interactions sociales; elle permet la stimulation et le défoulement d'un système de désirs bruts (les désirs individuels) opposé à un autre système tout aussi tributaire de la vitesse, celui des interactions sociales. Autrement dit, les cartoons renvoient à l'intransigeance des systèmes (économique, social, système de production et de consommation) les désirs que ceux-ci nient, écrasent, ignorent totalement et brutalement: désirs provoqués par le système lui-même, désirs de succès et de vie renouvelable, désirs de nourriture, de succès ou d'argent, envies, jalousies et vindications, etc.

Le public de Tex Avery est essentiellement composé de ce que l'on peut appeler le "prolétariat militaro-industriel». Outre le souvenir pénible et brutal de la Première Guerre mondiale (guerre absurde où les hommes sont réduits, dans tous les sens du terme, à la disette, l'attente, l'aveuglement et les besoins les plus bruts), c'est encore la crise économique et la quasiinstitutionnalisation du crime organisé durant le premier tiers $\mathrm{du} \mathrm{XX}^{\mathrm{e}}$ siècle, puis la mobilisation nationale autour de la Deuxième Grande Guerre, qui conduisent les cartoons vers un imaginaire où la brutalité des besoins coïncide avec le rêve de puissance, où l'excitation pour la nouveauté aboutit à la compulsion consommatrice.

La vitesse devient dans le cartoon l'expression de ces désirs, de leurs frustrations comme de leur sublimation. Ne pouvant pas toujours, pour des impératifs moraux et idéologiques, cautionner tous les désirs surgis à travers l'échec et les difficultés des interactions sociales, le cartoon va porter le système des désirs 
jusqu'au paroxysme de la vitesse. La vitesse produit l'illusion de l'assouvissement. Mais dans le monde des toons, tout est toujours à recommencer. Il n'y a pas de mort sans renaissance, et pas de surgissement dans le réel sans la dissolution presque subite mais temporaire de ce réel toujours dessiné en conformité avec l'immédiateté des désirs et la vitesse de leur sublimation.

Les cartoons donnent toujours à leurs protagonistes des moyens rêvés: les objets sont entièrement disponibles quand il s'agit de les prendre comme moyens, mais jamais entièrement accessibles dès qu'il s'agit de les concevoir comme fin. Dans l'escalade des événements et l'augmentation du rythme de l'activité "toonesque», il faut voir une matérialité diégétique qui s'oublie, qui pratique l'autodénégation en devenant malléable, soumise au gré des moindres caprices des protagonistes, toujours conforme à leurs petites mesquineries conjoncturelles: des marteaux apparaissent sous les manteaux, des pots de colle et des bâtons de dynamite sont disponibles à volonté au beau milieu du désert comme en pleine ville, des écriteaux surgissent de nulle part qui servent à berner l'adversaire et le conduire dans une mauvaise direction. L'accessoire apparaît alors souvent comme le prolongement du corps; rien ne le définit ni le démarque vraiment de la matière soi-disant vivante du corps ou de celle des décors. L'accessoire accède à l'univers "toonesque" telle la production même de l'activité du toon et de sa fantasmagorie. En cela, il est une preuve de "l'élasticité" du corps comique - pour employer l'expression utilisée par Olivier Mongin pour décrire le comique cinématographique ${ }^{3}$. La flexibilité et l'adaptabilité fantastiques des corps "toonesques" étonnent, mais la fin poursuivie par tout ce déploiement, ce but vers lequel est censé œuvrer l'arsenal des moyens "toonesques" est toujours inaccessible. L'inassouvissement reste la fin de tout, pour ne pas dire la non-fin nécessaire à la machine "toonesque». La variété, la profusion des moyens, des accessoires, des objets, le défilement sans limites des décors, rappellent que l'économie de production renvoie toujours le désir à sa perte, à son recommencement, projetant sans répit de nouveaux produits et suscitant des désirs toujours plus impérieux. L'univers de Tex Avery est celui où la matérialité et le contour des choses sont anéantis avant même que l'on ait le temps 
de les jauger. Le temps lui-même est sans cesse rattrapé par la fulgurance des désirs. L'activité "toonesque " est indissociable de la matérialité, de la fuite en avant de cette matérialité fantasmée sur la pellicule.

Le corps du toon appartient au même réseau d'activités et à la même fibre matérielle que la pellicule. Malgré la variété de ses mimiques qui expriment la peur, la douleur, l'émotion pure, malgré son exceptionnelle capacité à mimer l'épuisement, le corps du toon n'est jamais "significativement " fatigué, ne souffre jamais d'un épuisement qui mettrait en péril sa survie dans un univers où la violence est pourtant décuplée. Car la puissance de fragmentation, de pulvérisation et même de liquidation qui le frappe à tout instant n'est toujours qu'un moyen comme un autre de redynamiser le "vivant", de faire rebondir la situation. Fatigue, exaspération et essoufflement ne sont alors chez lui que l'expression d'un instant - l'expression de l'instantanéité à laquelle on prête ici, de manière ludique, une durée.

Ce temps que perdent les toons durant leurs élucubrations ne cesse de montrer, de dire en images qu'il ne vaut plus rien. Sous la gouverne d'une économie effrénée où le nouveau doit sans cesse se produire, le temps qui ne vaut que pour lui-même est un temps sans valeur. La série des Droopy est particulièrement sensible à la dévaluation du temps, à l'exaspération comique où conduit "la perte de temps" et l'accélération cauchemardesque. Reposant sur la logique de la compétition, la série des Droopy se caractérise par une incohérence diégétique, particulièrement à l'égard du déroulement temporel. La lenteur apparente de Droopy, petit chien taciturne, cache une fulgurante efficience, puisqu'il arrive toujours à devancer des adversaires manifestement déchaînés et qui, eux, se dépensent pourtant à grande vitesse et dans toutes les directions possibles. Ainsi en est-il d'un loup en fuite dans Dumb-Hounded (1943) qui use de tous les moyens de transport (automobile, bateau, train, motocyclette, avion, cheval...) et dont la course s'étend jusqu'au pôle Nord. Les limites du possible y explosent littéralement.

L'accélération n'est pas qu'un trait thématique du cartoon; il faut y entendre cette facilité avec laquelle l'univers "toonesque" glisse dans l'hyperbole. Le film Deputy Droopy (1955) met en 
scène deux malfaiteurs qui tentent de subtiliser l'argent gardé dans un coffre-fort chez le shérif. Adjoint du shérif, Droopy se voit confier la mission de garder le trésor et de donner l'alerte si un danger se présente. Après avoir fait ses recommandations, le shérif se dirige dans la pièce d'à côté en prévenant qu'il accourera au moindre bruit. Une fois cette règle du jeu instaurée, les deux bandits, couple burlesque typique composé d'un grand et d'un petit, s'engagent alors dans une escalade où, sans cesse déjoués par Droopy, ils auront à s'éloigner et revenir à répétition sur les lieux d'un crime qu'ils n'arrivent jamais à commettre. C'est qu'au lieu de les prendre simplement en chasse, Droopy multiplie les tours pour les obliger à faire du bruit. Contraints d'étouffer des bruits de toutes natures (des cris de douleur, des récriminations, des rires et même un rot, les deux vilains se voient dans l'obligation de sortir en alternance, à toute vitesse, du lieu où se trouve le coffre-fort. Chaque sortie implique un long parcours dans le désert où ils pourront libérer leurs cris et leurs bruits sans que personne ne les entende. Ces bruits deviennent ainsi objectifiables, dans la mesure où ils s'accumulent, sont enfermés dans des contenants, déplacés, déjetés comme des déchets. À répétition, les deux larrons sont déjoués pas le gardien du trésor: qu'ils mettent le pied sur des lames disposées à leur intention ou que l'un atteigne l'autre accidentellement avec son vilebrequin, chacun doit à chaque fois retenir son cri, sortir, courir dans le désert, crier, puis revenir. Les toons s'engagent dans un engrenage qui n'a pas de limite; Avery dessine pour eux une véritable chorégraphie où les corps et la matérialité, pris dans l'accélération de la compétition, subissent l'exacerbation du désir: des objets et des personnages-accessoires apparaissent soudain, tel un homard (au milieu du désert) dont Droopy se sert pour pincer ses adversaires, ou un biberon avec lequel il allaite le plus petit des deux ou encore ce téléphone qui surgit de derrière un rocher. Puis les langues des deux bandits vont s'étirer sur des kilomètres, leurs têtes seront interchangeables, le corps du grand ira courir dans le désert, dépourvu de tête, et le petit, resté chez le shérif, se retrouvera avec deux têtes plutôt qu'une.

Contrairement à certains films d'animation où les corps animés passaient par le procédé du rotoscope $\mathrm{e}^{4}$, les corps dessinés 
par Avery ne sont mimétiques qu’à travers les débordements de leurs désirs et les tics qu'imprime sur eux la gamme des émotions. Dans Little Rural Riding Hood (1949), le désir qu'éprouve le loup devant la pin-up déforme littéralement son corps, le désarticule: yeux qui rebondissent à des lieues, cou enroulé autour d'un objet, langue pendante s'étirant sous la table, membres élongés; c'est le corps tout entier qui bande à la vue d'une femme.

Le regard du voyeur, ces yeux qui décollent littéralement, nous renvoient à l'activité de prédation et au désir de dévoration qui prévalent chez le toon. Comme le rappelle Paul Virilio, l'invention relativement récente de la pin-up et du strip-tease ont tout à voir avec le divertissement militaire, mais aussi avec la transposition sur ce divertissement même des mœurs de la guerre. Ce que Virilio traduit par "l'éclaté cinématique" dans Guerre et cinéma recouvre parfaitement cette scène typique où, au découpage opéré par le sujet du regard sur le corps objectalisé de la pin-up, correspond la segmentation et même la pigmentation exagérée du corps du voyeur : "[...] le détail exagérément grossi [...]" de la pin-up par la "[...] perception hétérogène du voyeur militaire [...]": jambes, regard, croupe, etc.(p. 32-34) deviennent chez Avery le prétexte au grossissement et à l'hétérogénéité découpée du corps de son célèbre wolf.

Mais il y a encore un autre renvoi dans l'univers "toonesque" par lequel le corps retrouve le sens de la prédation et se rapproche de ses envies de dévoration. Si les toons sont les héritiers du burlesque comique et des corps-voyeurs pris dans une logique qui les dépasse, ils sont aussi les protagonistes parodiques des héros compétitifs, prédateurs et consommateurs de la littérature anglaise du XVIII ${ }^{\mathrm{e}}$ siècle, tels ceux de Swift, Richardson, Fielding et Defoe. Chez Avery, chaque héros est ramené à sa position dans la chaîne alimentaire. La question de l'intrigue devient alors facilement: qui mange qui? Pensons au film Who Killed Who? (1943) ou encore, plus explicitement, l'étrange What's Buzzin Buzzard (1943) ou deux oiseaux de proie attablés l'un devant l'autre, serviette de table au cou et fourchette à la main, entreprennent de se dévorer l'un l'autre. La rareté de la nourriture est un thème récurrent chez Avery. 
Parlant du corps du pauvre chez Defoe et Swift, Carol Houlihan Flynn emploie une expression qui laisse présager la folie des désirs en jeu chez Avery:
"[ [..] fictions of consumption $[\ldots]$ " : " [..] each [Defoe and Swift] turns to the figure of the cannibal to represent the failures of a society that devours its poor to maintain its consumptive growth. [...] While the consumption they delineate is a commercial result of civilization, the behavior of the consumers reveals its " savage» origins (p. 133).

Flynn voit Robinson Crusoe comme celui qui ordonne littéralement la chaîne alimentaire. Citant Defoe, il ajoute: «If we were but seen by the people of any other country, we would be taken for not cannibals, yet a sort of people that have a canine appetite" (p. 149). L'appétit canin est au cœur de l'univers "toonesque", sous sa forme dénotative comme sous ses dérivés connotatifs : le jeu du chat et de la souris, celui du chasseur et du canard. Le corps du toon est une version drolatique et violente tout à la fois de cet appétit thématisé chez Defoe; il exprime cet inassouvissement et la violence de la prédation qui l'accompagne. La matérialité participe d'une fuite en avant, prise à son tour dans la contrainte que subit l'individu pour sa survie et qui l'oblige à réinventer de toutes pièces son univers, à le construire à sa mesure, à produire sans cesse et à prévoir toujours ce que l'adversité réserve; c'est de ce désir qu'elle tire sa propension à la composition et à la recomposition. La matérialité " toonesque " n’a de réalité que la démesure des appétits auxquels elle est sujette. Si la peur du cannibalisme était associée au XVIII siècle à la peur du démembrement, à celle de la fragmentation du corps, à l'effrayante image d'un membre en train de rôtir sur le feu à quelques mètres des restes, la même imagerie déployée chez les toons ne peut que faire rire: c'est que la matérialité entière de cet univers semble infiniment dépeçable, digestible et recomposable.

Lîle de Crusoe prélude à l'univers du toon: un monde individualiste, construit de toutes pièces, simple, où les objets finissent par "apparaitre " par la force de la volonté (Crusoe) ou celle du fantasme (le toon). Un peu de poudre, de la nourriture, un décor rudimentaire, voilà l'univers déployé. Joué par le temps, 
mais capable de surmonter les accidents, le toon ne cesse néanmoins de déconstruire ce que Crusoe construit; si ce dernier refuse de se voir comme le jouet de la destinée et réclame sa pleine souveraineté de sujet, le toon ne cesse de miner sa condition de sujet-désirant en assumant le rôle de jouet. C'est ainsi qu'il se situe soit au-delà du sujet moderne (plus souverain encore dans sa capacité d'accepter de "jouer le jeu") soit en deçà (une instance au service des fantasmagories du Ça).

Cette capacité dont témoigne un corps à faire éclore l'univers tout entier, cette projection fantasmatique d'un appétit dont surgit un monde n'est cependant pas un trait appartenant exclusivement à l'imaginaire occidental. Elle trouve sa source aussi loin que dans les "tricksteries", ces tours joués par la figure archaïque du trickster. C'est que dans la continuité du burlesque, le comique d'animation renoue avec cette figure emblématique des traditions amérindiennes, avec les forces chtoniques et ce que Meletinsky appelle "le héros civilisateur", soit celui par qui le monde s'organise, ce créateur de désordre par lequel l'ordre s'instaure et la narrativité advient.

La zoomorphie du trickster est reprise par les toons, plus efficacement encore que ne le fait la fable animalière classique. Les ruses du trickster démiurge, ces tours joués par le corbeau sibérien ou le renard américain par exemple, sont toujours liés à la compétition alimentaire, à la quête de nourriture et à la tromperie induite par cette quête. La reprise du trickster opère d'ailleurs dans la comédie de la renaissance qui en emprunte les archétypes: gémellité, couples dépareillés, tours, prise en chasse, fuites et jeux de masques, etc. Le trickster, qui, pour le dire comme D. A. Beecher est "une expression de la rêverie de la liberté ", appartient à la logique du charivari et concentre ses activités autour de trois thématiques essentielles: la nourriture, le sexe et la violence 5 . Le toon doit donc au trickster son rapport particulier au corps et à la matérialité. Comme pour le trickster, le corps et la matérialité "toonesque" ne forment avec l'intrigue qu'une seule efficacité. C'est sur ce plan que régresse le toon par rapport au héros romanesque industrieux et impérialiste. Car la pulsion qui guide son activité corporelle ne correspond pas à ce que Peter Brooks décrit lorsqu'il parle de la narration du corps de la 
modernité occidentale et qu'il appelle "the epistemophilic project", c'est-à-dire "le désir de voir et de savoir ${ }^{6}$ ". Le désir du toon est en deçà de cette application à la raison ou de cette quête du savoir; ce qu'il veut, c'est prendre, manger, dévorer. L'œilprédateur, la caméra prédatrice à l'œuvre dans son univers répond plutôt à cet impératif brutal évoqué par Virilio lorsqu'il fait se rejoindre l'invention du cinéma et la logistique militaire (cartographie, cibles militaires, photographies aériennes, etc.). Mais si en effet les désirs et les frustrations des toons sont modelés sur les attentes du prolétariat militaro-industriel, cela n'empêche pas Avery de recourir aux "tricksteries " précisément pour remettre en question à la fois la construction moderne du corps social, sa sublimation par la maîtrise de l'optique et de l'espace, la souveraineté même de ce regard "épistemophilique" dont parle Peter Brooks.

Il est remarquable que le toon ait pu conserver, sinon même exposer de manière radicale, un trait important de sa nature chtonique : s'il agit tel un principe narratif (une loi du récit, un principe de l'économie intrigante, une clef logique), il est en même temps un "corps" propulsé par ses désirs et ses envies les plus naturelles. La concrétude du toon, sa matérialité évidente coïncident paradoxalement avec la dématérialisation constante de son univers et de son corps, avec la fragmentation fulgurante de ses désirs. La matérialité de ce corps filmique étant infiniment reconstituable, ne connaissant ni la mort ni la putréfaction, elle coïncide avec l'immatérialité du principe, avec l'air et la vitesse pure, avec le mouvement pur. Sur le modèle des forces chtoniques qui produisent l'univers, le néant semble produire quelque chose. Davatange ici que chez tout autre comique, Bergson est dépassé et Deleuze devrait ajouter: "[...] le comique n'est plus du mécanique plaqué sur du vivant, mais du mouvement de monde emportant et aspirant le vivant" (p. 89). Comme le faisait sur l'horizon mythologique le cataclysme des forces chtoniques, la vitesse emporte le toon et son univers pour les reconstituer aussitôt, parce que le toon fait corps avec son univers: l'univers "toonesque" est l'expression du corps "toonesque", de sa fantasmagorie, de ses désirs et de ses obsessions alimentaires. C'est un monde contenu dans une bouche. 
Très peu de films, sinon aucun, n'auront réussi à jouer, comme c'est le cas chez Tex Avery, sur la double valeur de la corporalisation et de la décorporalisation d'une manière aussi radicale, critique et lucide. S'accommodant d'une certaine légèreté, mais ne cédant pas à la facilité, c'est sous le couvert d'un divertissement de masse que l'univers "toonesque" s'est imposé à l'imaginaire, non pas comme une entité purement cinématographique, puisque le cartoon n'a pas le réel comme "prise de vue», ni comme pure "littérarité ", puisque sa narrativité n'a pas le texte pour support, mais plutôt comme une "activité" par laquelle le corps est sans cesse interpellé et les désirs convoqués dans un cadre fictionnel et critique franchement renouvelé.

\section{Université de Montréal}

\section{NOTES}

1 C'est le cas par exemple dans Screwball Squirrel (Avery, 1944), où un trou dans un arbre devient mobile et où, défiant les lois de la physique, des objets de grandes dimensions sont cachés derrière d'autres objets plus petits.

2 Il y aurait beaucoup à dire sur l'auto-ironisation de Avery qui, travaillant en vase clos avec son équipe de dessinateurs et de monteurs, avait l'habitude de se dessiner lui-même en train de travailler et de produire. L'équipe de Avery avait imaginé un véritable système d'autodérision pour se moquer des exigences de production et de standardisation d'Hollywood (l'usine à gags conçue sur le modèle de la production de Taylor). Jean-Pierre Berthomé raconte dans Tex Avery. La folie du cartoon qu'un animateur " $[\ldots]$ avait mis en place tout un système électrique qui allumait une petite lampe sous le bureau de chaque animateur. Dès que Ray [le directeur de production] descendait, le gars pressait un bouton et toutes les lampes s'allumaient, et tous les gars sautaient sur leur travail. Tous sauf les scénaristes et les réalisateurs qui travaillaient dans des bureaux voisins [qui] arrêtaient le boulot. En un an et demi, jamais Ray n'a trouvé un réalisateur ou un scénariste au travail [...] " (Paris: Artefact, 1986, p. 9).

3 C'est dans ces termes qu'Olivier Mongin parle du corps à corps qu'implique la situation comique chez Tati : "[...] le comique résidant alors dans l'élasticité d'un corps à corps plus ou moins tendu ou détendu, l'accessoire est toujours secondaire [...]" (p. 128).

4 Le rotoscope, inventé par Max Fleischer en 1915, permet de redessiner un corps réel, celui d'un acteur ou d'une actrice par exemple, en prise de vue réelle. La technique était surtout utilisée pour rendre les girls conformes à la sensualité attendue et réelle des danseuses professionnelles. Malgré l'introduction des girls dans les films de Avery, celui-ci n'a jamais fait appel au rotoscope. Ses girls sont certainement les seuls personnages chez Avery à échapper aux conditions d'élasticité et de fragmentation du toon. La technique du rotoscope, même imitée (certains dessins de Disney par exemple) convie à la chorégraphie d'un corps essentiellement mimétique, un corps dénué de vitesse et qui tend vers le mouvement en temps réel. 
5 Dans son analyse de la reprise du trickster dans la comédie anglaise du $\mathrm{XVII}^{\circ}$ siècle, Beecher caractérise ce dernier, comme le faisait Jung avant lui, comme un "hors-la-loi ". Mais surtout, il montre combien le trickster est amené à jouer un rôle prépondérant comme planificateur d'intrigues, se substituant même parfois à l'auteur comme instance de contrôle sur l'action. Sans aucun doute, des toons tels Daffy Duck ou Bugs Bunny doivent être ramenés à cette figure du trickster: "The false face, the calculated prevarication is the characterizing gesture of the trickster and the basis of his gaming tactics. The lie, from the quibble to the sophistic assault, serves to bring the other characters into his sphere of power.[...] the trickster is, above all, a connaisseur of his own wit" (D.A. Beecher, "Intriguers and Tricksters: The Manifestation of an Archetype in the Comedy of Renaissance", Revue de littérature comparée, $\mathrm{n}^{\circ} 1$, 1987, p. 17).

6 " [...] bodies emblazoned with meaning within the field of desire, desire that is originally and always, with whatever sublimations, sexual, but also by extension the desire to know [...]" (Peter Brooks, Body Work, Cambridge: Harvard University Press, 1993, p. 5).

\section{OUVRAGES CITÉS}

Beecher, D. A. "Intriguers and Tricksters: The Manifestations of an Archetype in the Comedy of Renaissance ". Revue de littérature comparée, n" 1 (1987) p. 5-29.

Berthomé, Jean-Pierre. Tex Avery. La folie du cartoon. Paris: Artefact, 1986.

Brion, Patrick, Tex Avery. Les dessins. Paris: Nathan Image, 1988.

Brooks, Peter. Body Work. Cambridge: Harvard University Press, 1993.

Deleuze, Gilles. L'Image-temps. Cinéma 2. Paris: Minuit, 1985.

Flynn, Carol Houlihan. The Body in Swift and Defoe. Cambridge: Cambridge University Press, 1990.

Furetière, Antoine. Le Roman bourgeois. Paris: Librairie Jules Talandier, 1666.

Mongin, Olivier, "Les métamorphoses du corps comique", Communications, n" 56 (1993), p. 125-138.

Virilio, Paul. Guerre et Cinéma I. Logique de la perception. Paris: Cahiers du cinéma, 1991.

\section{FILMOGRAPHIE}

Les films de Tex Avery:

Chez Warner Bros: Daffy Duck and Egghead (1937), Cinderella meats fella (1938), A Wild Hare (1940), All this and rabitt stew (1941).

Chez M. G. M.: Dumb-Hounded (1943), Red Hot Riding Hood (1943), Who Killed Who? (1943), What's buzzin buzzard (1943), Screwball Squirrel (1944), The Shooting of Dan McGoo (1945), Swing Shift Cinderella (1945), Hound Hunters (1947), Uncle Tom's Cabana (1947), King Size Canari (1947), The Cat That Hated People (1948), Little Rural Riding Hood (1949), The Chump Champ (1950). 